\title{
Costs and consequences of the Family Nurse Partnership \\ (FNP) programme in England: evidence from the Building
}

\section{Blocks trial [version 1; peer review: 2 approved]}

\author{
Kerry Bell(D1, Belen Corbacho (D1), Sarah Ronaldson (D1), Gerry Richardson2, \\ Kerry Hood 3 , Julia Sanders ${ }^{4}$, Michael Robling 5 , David J. Torgerson (D1, \\ Building Blocks Trial Group \\ ${ }^{1}$ Health Sciences, University of York, UK, York, North Yorkshire, YO10 5DD, UK \\ ${ }^{2}$ Centre for Health Economics, University of York, UK, York, North Yorkshire, YO10 5DD, UK \\ ${ }^{3}$ Centre for Trials Research, Cardiff University, Cardiff, CF14 4YS, UK \\ ${ }^{4}$ School of Healthcare Sciences, Cardiff University, Cardiff, CF14 4XN, UK \\ ${ }^{5}$ Population Health Trials, Cardiff University, Cardiff, CF14 4ER, UK
}

V1 First published: 13 Sep 2019, 8:1640

https://doi.org/10.12688/f1000research.20149.1

Latest published: 13 Sep 2019, 8:1640

https://doi.org/10.12688/f1000research.20149.1

\section{Abstract}

Background: The Family Nurse Partnership (FNP) is a licensed intensive home visiting intervention programme delivered to teenage mothers which was originally introduced in England in 2006 by the Department of Health and is now provided through local commissioning of public health services and supported by a national unit led by a consortium of partners. The Building Blocks (BB) trial aimed to explore the effectiveness and cost-effectiveness of this programme. This paper reports the results of an economic evaluation of the Building Blocks randomised controlled trial (RCT) based on a cost-consequence approach.

Methods: A large sample of 1618 families was followed-up at various intervals during pregnancy and for two years after birth. A costconsequence approach was taken to appraise the full range of costs arising from the intervention including both health and social measures of cost alongside the consequences of the trial, specifically, the primary outcomes.

Results: A large number of potential factors were identified that are likely to attract additional costs beyond the implementation costs of the intervention including both health and non-health outcomes. Conclusion: Given the extensive costs and only small beneficial consequences observed within the two year follow-up period, the cost-consequence model suggests that the FNP intervention is unlikely to be worth the substantial costs and policy makers may wish to consider other options for investment.

Trial registration: ISRCTN23019866 (20/04/2009)

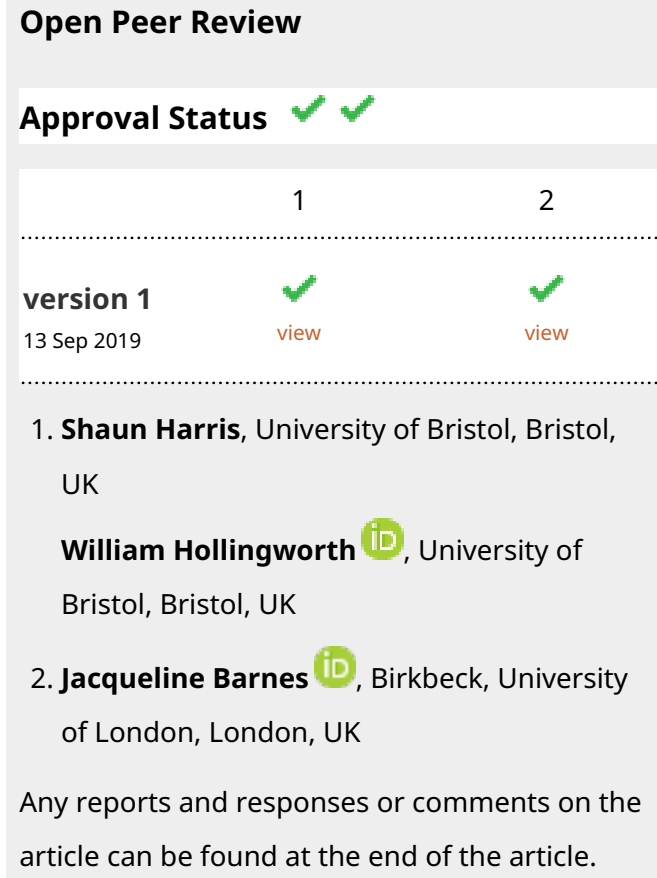

$\checkmark$

1. Shaun Harris, University of Bristol, Bristol, UK William Hollingworth ${ }^{1}$, University of Bristol, Bristol, UK 


\author{
Keywords \\ Randomised controlled trial, Cost-consequence analysis, Pregnancy in \\ adolescence, Prenatal care, Maternal health, Home visiting
}

Corresponding author: Kerry Bell (kerry.bell@york.ac.uk)

Author roles: Bell K: Conceptualization, Formal Analysis, Methodology, Writing - Original Draft Preparation, Writing - Review \& Editing; Corbacho B: Conceptualization, Formal Analysis, Investigation, Methodology, Writing - Review \& Editing; Ronaldson S:

Conceptualization, Methodology, Writing - Review \& Editing; Richardson G: Conceptualization, Methodology, Writing - Review \& Editing;

Hood K: Conceptualization, Methodology, Writing - Review \& Editing; Sanders J: Conceptualization, Investigation, Methodology, Project

Administration, Writing - Review \& Editing; Robling M: Conceptualization, Data Curation, Funding Acquisition, Investigation,

Methodology, Supervision, Writing - Review \& Editing; Torgerson DJ: Conceptualization, Methodology, Supervision, Writing - Review \& Editing;

Competing interests: No competing interests were disclosed.

Grant information: This work was supported by the Department of Health Policy Research Programme [006/0060]. The views and opinions expressed therein are those of the authors and do not necessarily reflect those of the UK Department of Health or the UK National Health Service.

The funders had no role in study design, data collection and analysis, decision to publish, or preparation of the manuscript.

Copyright: @ 2019 Bell K et al. This is an open access article distributed under the terms of the Creative Commons Attribution License, which permits unrestricted use, distribution, and reproduction in any medium, provided the original work is properly cited.

How to cite this article: Bell K, Corbacho B, Ronaldson S et al. Costs and consequences of the Family Nurse Partnership (FNP) programme in England: evidence from the Building Blocks trial [version 1; peer review: 2 approved] F1000Research 2019 , 8:1640 https://doi.org/10.12688/f1000research.20149.1

First published: 13 Sep 2019, 8:1640 https://doi.org/10.12688/f1000research.20149.1 


\section{Introduction}

The Building Blocks trial evaluated the Family Nurse Partnership (FNP) programme, an intensive, nurse-led home visiting programme for young, first time parents who live in areas with a low socio-economic profile. This programme was developed in the USA and was introduced in England by the Department of Health in 2006 with the aim of improving outcomes for health, wellbeing and social circumstances of young first-time mothers and their children. In October 2015 the FNP was transferred from NHS England to Local Authorities (LAs) and it is now provided in approximately 125 different LAs in England. The FNP programme was introduced to be an integral part of the progressive universalism approach recommended in The Healthy Child Programme (HCP). The HCP is delivered by the Family Nurse rather than by health visitors for women who enrol onto the programme.

Despite an extensive evidence base in the US, very little was known about the generalisability to a UK context which implements a very different health care model to that in the US. Hence, the Building Blocks (BB) trial was commissioned to evaluate the effectiveness and cost-effectiveness of the FNP intervention when delivered in a comprehensive publicly funded health care setting. The results from the effectiveness analysis showed no statistically or clinically significant difference associated with FNP for any of the four primary outcomes: smoking cessation (adjusted OR 0.90, 97.5\% CI 0.64-1.2), birth weight (adjusted mean difference $20.75 \mathrm{~g}, 97.5 \% \mathrm{CI}-47.73$ to 89.23 ), second pregnancies within two years (AOR 1.01, 0.77-1.33), or child A\&E attendances and admissions to hospital (AOR 1.32, $97.5 \%$ CI $0.99-1 \cdot 76, \mathrm{p}=0.03)^{1}$.

NICE has long recommended the use of cost-utility analysis as the primary means of assessing cost-effectiveness where health is the sole or dominant benefit of influence. However, with the move to local authority responsibility in 2013, NICE broadened its approach to the appraisal of public health interventions to place more emphasis on cost-consequence and cost-benefit analyses to supplement the cost-utility analysis for complex interventions ${ }^{2}$. Unlike the cost-utility analysis which reports only on the number and cost of QALYs gained, the cost-consequences analysis (CCA) is able to present disaggregated costs and a range of outcomes which allows readers to form their own conclusions on relevance and relative importance to their own decision making context ${ }^{3}$. This is particularly valuable for evaluation interventions with an array of health and non-health benefits that cannot be measured in a common unit. CCAs are not restricted to a particular viewpoint hence decision makers can estimate the impact of their decisions on sectors beyond health, such as education and criminal justice ${ }^{4}$. Furthermore, CCA is able to take many items into account that local authorities are likely to find important, including the trade-off between long-term goals and a paucity of short-run funding, and spill over effects into other areas of local government responsibility.
Given the complexity of the FNP programme and the diverse range of policy relevant outcome measures, a multi-faceted approach was taken to evaluate the programme's cost-effectiveness. A cost-utility analysis conducted alongside the trial showed only nominal benefits in terms of maternal QALY gains which were not considered cost-effective based on NICE (National Institute for Health and Care Excellence) acceptability thresholds ${ }^{5}$.

The analysis aims to list all relevant health and non-health related resource use, largely public or third sector providers of universal and specialist services locally accessible to teenagers of young children, and the costs associated with each of the trial arms as well as the consequences of the trial, specifically, the primary outcomes. In the UK, health care policy decisions are largely driven by the National Institute for Health and Care Excellence (NICE) which base their recommendations on the number of quality adjusted life years (QALYs) that can be gained by an intervention. As the children in the participating families were too young to draw QALY estimates, the overarching perspective of the wider economic analysis that was conducted alongside the trial could only be completed from the perspective of the mother due to availability of data. In keeping with this approach, the cost-consequence analysis also considered only resource use of the mother.

\section{Methods}

\section{Sample}

The Building Blocks trial was a two-arm pragmatic, nonblinded, parallel-group, randomised controlled trial (RCT) which recruited within a community midwifery setting at 18 partnerships between LAs and primary and secondary care organisations in England. A total of 1645 participants were randomised in the study. The present analysis is based on the 1618 participants recruited to the trial that did not later become ineligible (e.g. due to miscarriage) or withdraw their consent. Participants in the Intervention arm $(n=808)$ received home visits from the FNP nurse during their pregnancy and in the two years following childbirth. Participants allocated to the usual care arm $(n=810)$ received care from the local maternity and health visiting services in line with usual practice. Details on recruitment and follow-up rates are described in full elsewhere ${ }^{1}$. Informed consent was obtained from all individual participants included in the study.

\section{Outcome measures}

The primary outcome measures for the trial were tobacco use at late pregnancy (34-36 weeks' gestation), birth weight, emergency attendances and hospital admissions for the infant within 24 months of birth, and the proportion of women with a second pregnancy within 24 months post-partum. There were also a large number of secondary outcomes across a number of domains; socio-economic, maternal health and well-being, health behaviours, pregnancy and birth, social support and 
the use of services. For all primary outcomes a $97.5 \%$ confidence interval (CI) and p-value was presented; for all secondary outcomes a 95\% CI and p-value was presented. The outcome measures are described in full elsewhere ${ }^{1}$.

\section{Economic data collection}

Data for outcomes and resource use were collected by selfreported questionnaires at various time-points throughout the trial, specifically; baseline, late pregnancy (34-36 weeks gestation) and 6, 12, 18, and 24 months postpartum. Baseline and 24 month data were collected by face-to-face interview by a locally based researcher whilst data at other time points were collected via telephone by qualified telephone interviewers. Additionally, data related to the use of health care services for each trial participant were collected from Hospital Episode Statistics (HES) via NHS Digital and primary care (general practitioner (GP) records). Birth data were collected by records abstraction from maternity records.

\section{Resource use}

The mean numbers of the health and non-health care items per woman are presented for both groups for the duration of the trial in their respective units, e.g. mean number of GP visits, average number of weeks in education etc. The difference between the two groups for these items is also presented. The totals for each item (e.g. total number of GP visits) have been calculated for each participant in both groups over the duration of the trial (i.e. from baseline up to the 2-year interview).

Resource use is averaged across all women in each arm leading to small mean numbers for resource use. Median, minimum and maximum values are reported to highlight the highly skewed nature of resource use. Costs were largely associated with only a minority of women using each resource. Resource use and associated costs are reported in terms of the mother only. All consequences of the intervention, for both mother and child, are reported.

\section{Unit costs}

Unit costs applied to the resource use were retrieved from several sources. Unit costs pertaining to health care resource use (Table 1) were sourced from NHS reference costs ${ }^{6}$ and Personal Social Service Research Unit (PSSRU) Unit Costs of Health and Social Care 20137. However, as no unified document currently exists to provide unit costs for the non-health related items, these were sourced from other relevant sources such as governmental websites, research documents and reports. Given that costs were taken from a range of sources the years of pricing vary for each item. These are reported in Table 2. No attempt was made to discount/ inflate these items to a consistent rate as appropriate inflation rates for this type of resource are not available. It is thus acknowledged that current figures per resource use may differ slightly from those documented.

\section{Missing data}

For the purpose of the cost-consequence analysis, multiple imputation analysis was considered unnecessary as the model serves only to highlight likely resource use and the average costs per resource. This decision was further vindicated by the small number of women who reported using each of the resources, which in some cases were less than five women. Such small numbers of affirmative responses would prevent imputations from running effectively and result in high error rates. Instead, several assumptions were made to reduce the amount of missing data. Where a particular answer to a question meant that sub-questions were skipped, a value of zero was attributed to the sub-questions. For example, respondents were first asked a yes or no question as to whether they had utilised a particular resource, then how often following an affirmative response. Where respondents stated that they had not used a resource, frequency of use was assumed to be zero. Conversely, where a positive response was noted but not frequency value was given, a value of one was assumed as a conservative estimate. Due to the high number of variables and low number of complete cases, it was not considered pragmatic to summarise each variable by complete case and adjusted values.

\section{Statistical analysis}

The mean resource use was calculated for each resource use item according to the appropriate unit (e.g. mean number of days). The incremental difference between trial arms was calculated for each item of resource use by subtracting the values of participants allocated to receive usual care from the values of participants who were allocated to receive the intervention (FNP). Mean unit costs were calculated by item for each of the trial arms by multiplying the resource use by an appropriate unit costs (see Table 3 for unit costs). Resource use costs were summed by type, e.g. health related resource use or nonhealth related resource use. All analyses were conducted in Stata version $12^{8}$. The consequences presented in Table 3 are the primary outcomes of the Building Blocks trial and full details of how these were calculated are presented in primary trial paper?

\section{Results}

Unit costs of all health and non-health related resources used by the mother only are presented in Table 1 and Table 2. The results of the cost-consequences analysis can be seen in Table 3 . For each cost item, the mean resource use per participant for both the FNP arm and the usual care arm are displayed, alongside the incremental resource use. The mean cost per participant is also shown for each item according to trial arm, as well as the incremental cost. In terms of the consequences, the primary outcomes of the Building Blocks trial are listed for both the FNP arm, usual care arm and the difference between the two. A descriptive summary of the costs and consequences is provided, whereby items are discussed in a disaggregated format for the broad range of costs and consequences (spanning health care and non-health care) included in the analysis. 


\begin{tabular}{|c|c|c|c|c|}
\hline Item & Unit & Cost & Reference & Notes \\
\hline GP & $\begin{array}{l}\text { Per Surgery consultation } \\
\text { lasting } 11.7 \text { min } \\
\text { Per out of surgery (home } \\
\text { visiting) lasting } 23.4 \text { min }\end{array}$ & $£ 114$ & $\begin{array}{l}\text { Unit Costs of Health } \\
\text { and Social Care } 2013\end{array}$ & Including direct care staff costs \& qualifications \\
\hline GP Nurse & $\begin{array}{l}\text { Per Surgery consultation } \\
\text { lasting } 15.5 \text { minutes } \\
\text { Per home visiting lasting } \\
23.4 \text { min }\end{array}$ & $\begin{array}{l}£ 13.4 \\
£ 27.3\end{array}$ & $\begin{array}{l}\text { Unit Costs of Health } \\
\text { and Social Care } 2013\end{array}$ & Assume same duration than GP home visit \\
\hline Midwife & $\begin{array}{l}\text { Antenatal visit } \\
\text { (Community) } \\
\text { Postnatal visit } \\
\text { (Community) } \\
\text { Home visit } \\
\text { Midwife episode }\end{array}$ & $\begin{array}{l}£ 51 \\
£ 68 \\
£ 70 \\
£ 65\end{array}$ & $\begin{array}{l}\text { NHS reference costs } \\
2012 / 2013 \\
\text { (NHS trusts and NHS } \\
\text { foundation trusts) } \\
\text { Unit Costs of Health } \\
\text { and Social Care } 2013\end{array}$ & $\begin{array}{l}\text { Community Health Services - Health Visiting and } \\
\text { Midwifery } \\
\text { Total Outpatient attendances data }\end{array}$ \\
\hline Health visitor & $\begin{array}{l}\text { Per hour } \\
\text { Per hour of home visiting }\end{array}$ & $\begin{array}{l}£ 49 \\
£ 71\end{array}$ & $\begin{array}{l}\text { Unit Costs of Health } \\
\text { and Social Care } 2010\end{array}$ & Assume same duration than GP home visits \\
\hline Counsellor & Surgery consultation & $£ 58$ & $\begin{array}{l}\text { Unit Costs of Health } \\
\text { and Social Care } 2013\end{array}$ & \\
\hline Mental health & Per hour per team member & $£ 36$ & $\begin{array}{l}\text { Unit Costs of Health } \\
\text { and Social Care } 2013\end{array}$ & $\begin{array}{l}\text { Community mental health team for adults with } \\
\text { mental health problems. }\end{array}$ \\
\hline $\begin{array}{l}\text { Crisis } \\
\text { Resolution team }\end{array}$ & Per hour per team member & $£ 37$ & $\begin{array}{l}\text { Unit Costs of Health } \\
\text { and Social Care } 2013\end{array}$ & \\
\hline Support worker & Per hour & $£ 22$ & $\begin{array}{l}\text { Unit Costs of Health } \\
\text { and Social Care } 2013\end{array}$ & \\
\hline Social worker & Per hour & $£ 79$ & $\begin{array}{l}\text { Unit Costs of Health } \\
\text { and Social Care } 2013\end{array}$ & \\
\hline Physiotherapist & $\begin{array}{l}\text { Surgery session per hour } \\
\text { Hospital session per hour }\end{array}$ & $\begin{array}{l}£ 34 \\
£ 36\end{array}$ & $\begin{array}{l}\text { Unit Costs of Health } \\
\text { and Social Care } 2013\end{array}$ & \\
\hline $\begin{array}{l}\text { FNP Supervisor } \\
\text { Nurse }\end{array}$ & $\begin{array}{l}\text { Clinic or phone visit } \\
\text { per minute } \\
\text { Home visit per minute }\end{array}$ & $\begin{array}{l}£ 1.34 \\
£ 1.62\end{array}$ & $\begin{array}{l}\text { Unit Costs of Health } \\
\text { and Social Care } 2013\end{array}$ & $\begin{array}{l}\text { Qualified nursing, midwifery \& health visiting staff by } \\
\text { Agenda for change band } 8 \mathrm{a} \text {, NHS England. } \\
\text { Ratio of direct time on: } \\
\text { Home visits }(1: 0.45) \\
\text { Patient work }(1: 0.20)\end{array}$ \\
\hline FNP Nurse & $\begin{array}{l}\text { Clinic or phone visit } \\
\text { per minute } \\
\text { Home visit per minute }\end{array}$ & $\begin{array}{l}£ 1.17 \\
£ 1.41\end{array}$ & $\begin{array}{l}\text { Unit Costs of Health } \\
\text { and Social Care } 2013\end{array}$ & $\begin{array}{l}\text { Qualified nursing, midwifery \& health visiting staff by } \\
\text { Agenda for change band } 7 \text {, NHS England. } \\
\text { Home visits }(1: 0.45) \\
\text { Patient work }(1: 0.20)\end{array}$ \\
\hline
\end{tabular}

$\wedge$ PSSRU unit costs are based on mean full-time basic salary; including overheads and qualifications.

FNP - Family Nurse Partnership, PSSRU - Personal Social Service Research Unit 
Table 2. Unit costs for non-health resource use.

\begin{tabular}{|c|c|c|c|c|}
\hline \multicolumn{5}{|c|}{ Non-health related resource use } \\
\hline \multicolumn{5}{|l|}{ Education } \\
\hline $\begin{array}{l}\text { Mainstream school or } \\
\text { further education (FE) } \\
\text { college }\end{array}$ & $\begin{array}{l}\text { Per week of } \\
\text { education }\end{array}$ & $£ 77$ & $\begin{array}{l}\text { The Centre for Social Justice (Press release, } \\
\text { 2011) }\end{array}$ & $\begin{array}{l}\text { Cost of mainstream education cited } \\
\text { at } £ 4000 \text { per child per year. This was } \\
\text { divided by } 52 \text { to give an estimate of } \\
\text { weekly rate }\end{array}$ \\
\hline $\begin{array}{l}\text { Learning support unit } \\
\text { (LSU) }\end{array}$ & $\begin{array}{l}\text { Per week of } \\
\text { education }\end{array}$ & $\begin{array}{l}£ 40 \\
(+£ 77)\end{array}$ & $\begin{array}{l}\text { North Lincolnshire Learning Support Unit, } \\
2012 \text { (http://shareit.yhgfl.net/nlincs/cpd/ } \\
\text { ?page_id=2159) }\end{array}$ & $\begin{array}{l}\text { Cost per session of } 1 \text { centre. Assumed } \\
\text { mothers would have at least } 1 \text { session } \\
\text { per week in addition to regular } \\
\text { schooling }\end{array}$ \\
\hline $\begin{array}{l}\text { Pupil referral unit } \\
\text { (PRU) }\end{array}$ & $\begin{array}{l}\text { Per week of } \\
\text { education }\end{array}$ & $£ 346.15$ & $\begin{array}{l}\text { The Centre for Social Justice (Press release, } \\
\text { 2011) }\end{array}$ & $\begin{array}{l}\text { Cost of PRU education cited at } £ 18,000 \\
\text { per child per year. This was divided by } \\
52 \text { to give an estimate of weekly rate }\end{array}$ \\
\hline $\begin{array}{l}\text { Teenage mums } \\
\text { support unit (TMSU) }\end{array}$ & $\begin{array}{l}\text { Per week of } \\
\text { education }\end{array}$ & $\begin{array}{l}£ 167.50 \\
(+£ 77)\end{array}$ & $\begin{array}{l}\text { Government published information published } \\
\text { online May, } 2014 \text { (https://www.gov.uk/16-to- } \\
\text { 19-education-financial-support-for-students) }\end{array}$ & $\begin{array}{l}\text { Education charged at the same rate } \\
\text { as mainstream schooling. Mothers in } \\
\text { a TMSU are eligible for 'Care to Learn' } \\
\text { funding which provides a bursary of } \\
£ 160-£ 175 \text { per week (average } £ 167.50 \text { ) }\end{array}$ \\
\hline \multicolumn{5}{|c|}{ Other supportive services } \\
\hline Connexions advisor & $\begin{array}{l}\text { Cost per } \\
\text { hour }\end{array}$ & $£ 38.50$ & Unit Costs of Health and Social Care 2013 & $\begin{array}{l}\text { Page } 217 \\
£ 847 \text { per } 22 \text { hours of a connexions } \\
\text { advisors time gives an hourly rate of } \\
£ 38.50 \text { per hour }\end{array}$ \\
\hline School nurse & $\begin{array}{l}\text { Unit of } \\
\text { activity }\end{array}$ & $£ 27$ & Unit Costs of Health and Social Care 2013 & $\begin{array}{l}\text { Average UK unit cost of school based } \\
\text { children's health services }\end{array}$ \\
\hline $\begin{array}{l}\text { Young people centre/ } \\
\text { youth service }\end{array}$ & $\begin{array}{l}\text { Unit of } \\
\text { activity }\end{array}$ & $£ 17$ & $\begin{array}{l}\text { The cost of providing street based youth work } \\
\text { in deprived } \\
\text { Communities, } 2004 \text { (Joseph Rowntree } \\
\text { Foundation Report) }\end{array}$ & $\begin{array}{l}\text { Cost per contact of youth services } \\
\text { project }\end{array}$ \\
\hline Children's centre & $\begin{array}{l}\text { Unit cost } \\
\text { per hour }\end{array}$ & $£ 64.25$ & $\begin{array}{l}\text { Cost Effectiveness in Sure Start Local } \\
\text { Programmes: } \\
\text { A Synthesis of Local Evaluation Findings, } \\
2005\end{array}$ & Unit cost of Sure Start children's centres \\
\hline $\begin{array}{l}\text { Child development } \\
\text { centre }\end{array}$ & $\begin{array}{l}\text { Unit cost } \\
\text { per hour }\end{array}$ & $£ 34$ & Unit Costs of Health and Social Care 2013 & Unit cost for key worker \\
\hline Crèche/ day nursery & $\begin{array}{l}\text { Cost per } \\
\text { hour }\end{array}$ & $£ 4.28$ & Range of current sources, 2014 & $\begin{array}{l}\text { Average cost across a range of crèche/ } \\
\text { day nursery facilities }\end{array}$ \\
\hline Toddler group & $\begin{array}{l}\text { Unit cost } \\
\text { per hour }\end{array}$ & $£ 5$ & Unit Costs of Health and Social Care, 2005 & Page 26, Unit cost of toddler group \\
\hline Leaving care services & $\begin{array}{l}\text { Cost per } \\
\text { hour }\end{array}$ & $£ 20$ & $\begin{array}{l}\text { Young People Leaving Care: A Study of Costs } \\
\text { and Outcomes, } 2006\end{array}$ & $\begin{array}{l}\text { Page } 175 \text {, Leaving care worker/ } \\
\text { personal adviser (per hour of client } \\
\text { related activity) }\end{array}$ \\
\hline Youth offending team & $\begin{array}{l}\text { Unit cost } \\
\text { per hour }\end{array}$ & $£ 29$ & $\begin{array}{l}\text { Unit Costs in Criminal Justice } \\
\text { (UCCJ), } 2013\end{array}$ & $\begin{array}{l}\text { Page 65, Cost of Youth Offending Team } \\
\text { (YOT) practitioner }\end{array}$ \\
\hline Social worker & $\begin{array}{l}\text { Unit cost } \\
\text { per hour }\end{array}$ & $£ 57$ & Unit Costs of Health and Social Care 2013 & $\begin{array}{l}\text { Page 197, Unit cost per hour of social } \\
\text { worker }\end{array}$ \\
\hline \multicolumn{5}{|l|}{ Childcare } \\
\hline $\begin{array}{l}\text { Crèche/ day nursery } \\
\text { at school or college }\end{array}$ & $\begin{array}{l}\text { Cost per } \\
\text { hour }\end{array}$ & $£ 4.28$ & Range of current sources, 2014 & $\begin{array}{l}\text { Average cost across a range of crèche/ } \\
\text { day nursery facilities }\end{array}$ \\
\hline $\begin{array}{l}\text { Nursery group at } \\
\text { children's centre }\end{array}$ & $\begin{array}{l}\text { Cost per } \\
\text { hour }\end{array}$ & $£ 4.40$ & Childcare Costs Survey, 2014 & $\begin{array}{l}\text { Page } 4 \text {, Average UK cost of } 25 \text { hours } \\
\text { per week £109.89 which equates to } \\
£ 4.40 \text { per hour }\end{array}$ \\
\hline Child-minder & $\begin{array}{l}\text { Cost per } \\
\text { hour }\end{array}$ & $£ 3.99$ & Childcare Costs Survey, 2014 & $\begin{array}{l}\text { Page } 4 \text {, Average UK cost of } 25 \text { hours } \\
\text { per week £99.77 which equates to } \\
£ 3.99 \text { per hour }\end{array}$ \\
\hline
\end{tabular}




\begin{tabular}{|c|c|c|c|c|}
\hline \multicolumn{5}{|c|}{ Non-health related resource use } \\
\hline Any other childcare & $\begin{array}{l}\text { Cost per } \\
\text { hour }\end{array}$ & $£ 3.82$ & Childcare Costs Survey, 2014 & $\begin{array}{l}\text { Page 4, Mean cost of all types of } \\
\text { childcare }\end{array}$ \\
\hline \multicolumn{5}{|l|}{ Foster care (weeks) } \\
\hline Foster care mother & $\begin{array}{l}\text { Unit cost } \\
\text { per week }\end{array}$ & $£ 636$ & Unit Costs of Health and Social Care, 2013 & $\begin{array}{l}\text { Page } 88 \text {, Unit cost per week of foster } \\
\text { care inclusive of admin and social } \\
\text { services }\end{array}$ \\
\hline \multicolumn{5}{|c|}{ Temporary accommodation } \\
\hline $\mathrm{B} \& \mathrm{~B}$ & $\begin{array}{l}\text { Cost per } \\
\text { week }\end{array}$ & $£ 334.95$ & $\begin{array}{l}\text { Immediate costs to government of loss of } \\
\text { home, } 2012\end{array}$ & Page 4, Average cost per week, UK \\
\hline $\begin{array}{l}\text { Teenage parent } \\
\text { accommodation }\end{array}$ & $\begin{array}{l}\text { Cost per } \\
\text { week }\end{array}$ & $£ 151.78$ & $\begin{array}{l}\text { Young people and teenage parent } \\
\text { accommodation based services and floating } \\
\text { support services, } 2010 \\
\text { Supported Housing Commissioning } \\
\text { Strategy - 2005-2010, } 2005\end{array}$ & $\begin{array}{l}\text { Average of } 2 \text { sites taken: } \\
\text { Page 4, Average cost per week (Brent, } \\
\text { London) } \\
\text { Page 29, Average cost per week } \\
\text { (Sheffield) }\end{array}$ \\
\hline $\begin{array}{l}\text { Supported } \\
\text { accommodation }\end{array}$ & $\begin{array}{l}\text { Cost per } \\
\text { week }\end{array}$ & $£ 150$ & $\begin{array}{l}\text { Supported lodgings as a housing option for } \\
\text { young people report, } 2008\end{array}$ & Page 32, Average cost per week, UK \\
\hline $\begin{array}{l}\text { Mother and baby } \\
\text { hostel/unit }\end{array}$ & $\begin{array}{l}\text { Cost per } \\
\text { week }\end{array}$ & $£ 230$ & $\begin{array}{l}\text { Christian Family Concern Mother \& Baby Unit, } \\
2014 \text { (http://www.familyspacecroydon.co.uk/ } \\
\text { Contacts/details/christian-family-concern- } \\
\text { mother-baby-unit/) }\end{array}$ & Actual cost per week at 1 site in London \\
\hline Women's refuge & $\begin{array}{l}\text { Cost per } \\
\text { week }\end{array}$ & $£ 421.15$ & The Cost of Domestic Violence, 2004 & $\begin{array}{l}\text { Page } 76 \text {, Average of UK women's } \\
\text { refuges }\end{array}$ \\
\hline Homeless hostel & $\begin{array}{l}\text { Cost per } \\
\text { week }\end{array}$ & $£ 107.45$ & $\begin{array}{l}\text { Immediate costs to government of loss of } \\
\text { home, } 2012\end{array}$ & Page 4, Average cost per week, UK \\
\hline
\end{tabular}

Table 3. Cost-consequence balance sheet.

\begin{tabular}{|c|c|c|c|c|c|c|}
\hline \multirow{3}{*}{ Costs (resource use) } & \multicolumn{3}{|c|}{$\begin{array}{l}\text { Mean resource use per } \\
\text { participant }\end{array}$} & \multicolumn{3}{|c|}{ Mean cost per participant } \\
\hline & FNP & $\begin{array}{l}\text { Usual } \\
\text { care }\end{array}$ & $\begin{array}{l}\text { Incremental } \\
\text { (FNP-Usual } \\
\text { Care) }\end{array}$ & FNP & Usual care & $\begin{array}{c}\text { Incremental } \\
\text { (FNP-Usual Care) }\end{array}$ \\
\hline & \multicolumn{6}{|c|}{ Health related resource use } \\
\hline \multicolumn{7}{|c|}{ Inpatient attendances (Length of stay/ number of day admittances) } \\
\hline Inpatient stay & 3.99 & 4.09 & -0.10 & 6354.58 & 6661.18 & -306.60 \\
\hline Day admittances & 3.53 & 3.58 & -0.05 & 775.22 & 781.73 & -6.51 \\
\hline \multicolumn{7}{|c|}{ Outpatient attendances (Number of attendances) } \\
\hline Maternity services & 7.31 & 7.13 & 0.17 & 733.13 & 716.18 & 16.95 \\
\hline Other attendances & 1.31 & 1.42 & -0.11 & 156.37 & 161.23 & -4.86 \\
\hline \multicolumn{7}{|c|}{ Hospital-related resource use (Number of attendances) } \\
\hline A\&E visit & 6.50 & 9.00 & -2.50 & 167.07 & 172.79 & -5.73 \\
\hline \multicolumn{7}{|c|}{ Community based resources use (Number of clinic attendances/ home visits) } \\
\hline Midwife visits & 10.40 & 10.69 & -0.28 & 622.91 & 643.87 & -20.97 \\
\hline Health visitor visits & 5.88 & 12.93 & -7.05 & 135.68 & 217.78 & -82.10 \\
\hline \multicolumn{7}{|c|}{ GP visitation (Number of visits) } \\
\hline Surgery visits & 9.36 & 8.46 & 0.90 & 421.35 & 380.54 & 40.80 \\
\hline Home-based visits & 0.21 & 0.21 & 0.00 & 24.31 & 24.45 & -0.14 \\
\hline \multicolumn{7}{|c|}{ Nurse visitation (Number of visits) } \\
\hline Surgery visits & 2.07 & 2.20 & -0.13 & 21.10 & 22.40 & -1.30 \\
\hline
\end{tabular}




\begin{tabular}{|c|c|c|c|c|c|c|}
\hline \multirow[b]{2}{*}{ Costs (resource use) } & \multicolumn{3}{|c|}{$\begin{array}{l}\text { Mean resource use per } \\
\text { participant }\end{array}$} & \multicolumn{3}{|c|}{ Mean cost per participant } \\
\hline & FNP & $\begin{array}{l}\text { Usual } \\
\text { care }\end{array}$ & $\begin{array}{l}\text { Incremental } \\
\text { (FNP-Usual } \\
\text { Care) }\end{array}$ & FNP & Usual care & $\begin{array}{c}\text { Incremental } \\
\text { (FNP-Usual Care) }\end{array}$ \\
\hline \multicolumn{7}{|c|}{ Non-health related resource use } \\
\hline \multicolumn{7}{|l|}{ Education } \\
\hline Mainstream education & 6.90 & 6.62 & 0.28 & 710.36 & 682.15 & 28.21 \\
\hline Learning support unit & 0.26 & 0.19 & 0.07 & 10.40 & 7.75 & 2.65 \\
\hline Pupil referral unit & 0.00 & 0.01 & -0.01 & 0.00 & 2.88 & -2.88 \\
\hline Teenage mother support unit & 0.26 & 0.29 & -0.03 & 67.63 & 79.15 & -11.52 \\
\hline \multicolumn{7}{|c|}{ Other supportive services (Number of contacts) } \\
\hline Connexions advisor & 1.51 & 1.38 & 0.13 & 58.13 & 53.23 & 4.90 \\
\hline School nurse & 0.04 & 0.04 & 0.00 & 3.41 & 4.10 & -0.69 \\
\hline Youth services & 0.16 & 0.23 & -0.07 & 2.76 & 3.84 & -1.08 \\
\hline Family information service & 0.08 & 0.13 & -0.05 & 2.65 & 4.45 & -1.80 \\
\hline Children's Centre & 2.31 & 1.96 & 0.35 & 148.30 & 125.64 & 22.66 \\
\hline Child development centre & 0.02 & 0.10 & -0.08 & 0.84 & 3.23 & -2.39 \\
\hline Crèche/day nursery & 0.48 & 0.49 & -0.01 & 2.04 & 2.09 & -0.05 \\
\hline Toddler group & 0.93 & 0.83 & 0.10 & 4.63 & 4.17 & 0.46 \\
\hline Leaving care service & 0.08 & 0.06 & 0.02 & 1.53 & 1.28 & 0.25 \\
\hline Fostering services & 0.01 & 0.05 & -0.04 & 0.52 & 1.89 & -1.37 \\
\hline Youth offending team & 0.05 & 0.01 & 0.04 & 1.51 & 0.18 & 1.33 \\
\hline Social worker & 0.72 & 0.64 & 0.08 & 28.86 & 25.58 & 3.28 \\
\hline Addiction support unit & 0.01 & 0.01 & 0.00 & 0.35 & 0.35 & 0.00 \\
\hline \multicolumn{7}{|l|}{ Childcare (Days per week) } \\
\hline Crèche / Day nursery at school or college & 1.35 & 1.10 & 0.25 & 5.79 & 4.71 & 1.08 \\
\hline Nursery group at Children's Centre & 0.38 & 0.25 & 0.13 & 1.68 & 1.09 & 0.59 \\
\hline Child-minder & 0.46 & 0.34 & 0.12 & 1.85 & 1.35 & 0.50 \\
\hline Any other childcare & 0.88 & 0.77 & 0.11 & 3.38 & 2.96 & 0.42 \\
\hline \multicolumn{7}{|l|}{ Foster care (Weeks) } \\
\hline Mother & 0.10 & 0.18 & -0.08 & 60.61 & 114.64 & -54.03 \\
\hline Child & 0.18 & 0.17 & 0.01 & 117.28 & 106.00 & 11.28 \\
\hline \multicolumn{7}{|l|}{ Housing (Weeks) } \\
\hline $\mathrm{B} \& \mathrm{~B}$ & 0.22 & 0.26 & -0.04 & 75.03 & 87.67 & -12.64 \\
\hline Teenage parent accommodation & 0.74 & 0.36 & 0.38 & 146.21 & 70.48 & 75.73 \\
\hline Supported accommodation & 0.75 & 0.39 & 0.36 & 112.13 & 57.78 & 54.35 \\
\hline Mother \& baby hostel/unit & 1.07 & 0.89 & 0.18 & 246.51 & 204.44 & 42.07 \\
\hline Women's refuge & 0.04 & 0.11 & -0.07 & 15.12 & 46.27 & -31.15 \\
\hline Homeless hostel & 0.34 & 0.36 & -0.02 & 36.57 & 38.34 & -1.77 \\
\hline \multicolumn{7}{|c|}{ Consequences } \\
\hline \multicolumn{4}{|c|}{ Smoking (smoking or non-smoking in late pregnancy) } & \multicolumn{3}{|c|}{ OR: 0.9 [97.5\% Cl: 0.67 to 1.22$] p=0.51$} \\
\hline \multicolumn{4}{|c|}{ Birth weight (mean difference in grams) } & \multicolumn{3}{|c|}{$\begin{array}{l}\text { Mean adjusted difference }=20.75 \mathrm{~g} \\
{[97.5 \% \mathrm{Cl}:-39.13 \text { to } 80.64] \mathrm{p}=0.497}\end{array}$} \\
\hline \multicolumn{4}{|c|}{$\begin{array}{l}\text { Child A\&E attendances and admissions (attendance/admission or no } \\
\text { attendance/admission) }\end{array}$} & \multicolumn{3}{|c|}{$\begin{array}{l}\text { OR: } 1.32[97.5 \% \mathrm{Cl}: 1.02 \text { to } 1.70] \\
p=0.033\end{array}$} \\
\hline \multicolumn{4}{|c|}{$\begin{array}{l}\text { Short duration to subsequent pregnancy (<2years) (pregnancy or no } \\
\text { pregnancy) }\end{array}$} & \multicolumn{3}{|c|}{$\begin{array}{l}\text { OR: } 1.01[97.5 \% \mathrm{Cl}: 0.80 \text { to } 1.28] \\
p=0.920\end{array}$} \\
\hline
\end{tabular}

FNP - Family Nurse Partnership 


\section{Costs}

Little difference was observed in terms of average health care resource use across the majority of services though consistently small differences were found between trial arms in favour of FNP. The largest differences in terms of resource use were the average number of $\mathrm{A} \& \mathrm{E}$ attendances and the mean number of health visitor visits received, both of which, as anticipated, were lower for women receiving the FNP intervention compared to women under usual care.

As only small differences in average resource use were observed, the average per woman spend on each resource reflected only slightly notable differences. Overall, average total health-related resource use costs were slightly lower for the FNP arm compared to usual care. Lower average costs were found in the FNP arm in relation to inpatient care, outpatient attendances not related to maternity services, A\&E attendances, and midwife and health visitor visitations. The largest difference in costs was found in relation to inpatient care requiring one or more nights in hospital. In this instance, the mean difference between arms was $£ 307$ with lower costs being associated with FNP recipients. A difference was also found between the average cost per participant in terms of community-based maternity and early years services, specifically health visitor and midwife visitation. On average £82 less was spent per woman receiving the FNP intervention compared to usual care for health visitor visits (both home and clinic based) whilst $£ 21$ less was spent per woman on midwife visits (both home and clinic based) reflecting a mean reduction in spend of just over $£ 100$ per woman on these key early years services. The number of health visitor visits received by women was lower in the FNP arm than the usual care arm as visits from FNP nurses were received in place of standard health visitor visits. Consequently, though the cost attributed to health visitor visits appears reduced, there is the additional cost of the FNP nurses. The cost of the health visitors provided in usual care would thus not be saved through the FNP intervention but would be spent instead on specific FNP trained nurses. Only minor differences were noted in terms of cost associated with inpatient day admittances not requiring an over-night stay (-£6.51), nonmaternity based outpatient attendances (-£4.86), and A\&E attendances (-£5.73), with all being in favour of FNP. Omitting the intervention costs, a total of $£ 8,872$ an average was spent on health care for women receiving the FNP intervention compared to a total average of $£ 9,162$ per woman in the usual care arm, a difference of $£ 290$ per woman between the two arms.

In addition to health care resource use, there was very little difference in the non-health care resource use between the two groups. The average cost of non-health related resource use for women receiving the FNP intervention was $£ 1,866$ compared to $£ 1,738$ for women under usual care, an average difference of only $£ 128$ per woman in favour of the control group. In general, reported uptake of all non-health related resources was fairly low in both groups as can be seen through the mean and median values presented in Table 3. For the majority of resource items, the median resource use for both groups is zero, confirming a low uptake. Mean values are thus influenced by only a minority of women.

Participation in education and training was also similar between groups for both mainstream schooling and dedicated support units with little difference in resource use or mean costs.

In terms of supportive services, children's centres, such as Sure Start centres, were the most widely used service by both groups, though this averaged at only 2 visits per woman in each of the trial arms. Mean costs of children's centre use was slightly higher in the FNP arm. This is likely a consequence of outlying values as the maximum number of visits was considerably higher in the FNP arm compared to usual care (38 and 29).

Resource use of childcare was found to be particularly low at all time-points and overall mean resource use per woman, within both groups, was particularly low. Slightly higher costs were found in FNP women in line with the marginally higher rates of resource use. The most widely used type of childcare was crèche/day nursery within a school or college though the mean number of weeks used throughout the follow-up period was very low at only 1.35 weeks for FNP women and 1.10 weeks for usual care women. As this resource use was low the total resource use was low resulting in a low average cost per woman of $£ 5.79$ per FNP woman and $£ 4.71$ per usual care woman though the cost for women actually using childcare would be much higher.

Resource use for foster care and temporary accommodation was also low in terms of the number of women reporting usage of these resources for both groups of women. Average costs are thus driven by a minority of women. Average costs for temporary accommodation were higher for FNP women than usual care women for teenage parent accommodation, supported accommodation and mother and baby units due to increased use of these by FNP women. The largest average difference between groups was for teenage parent accommodation, with FNP women costing on average $£ 75.73$ more than usual care women; again this was due to higher levels of resource use in the FNP group.

\section{Consequences}

Primary outcomes. No difference was observed in the rate of smoking in late pregnancy between the two arms (adjusted OR: $0.90,97.5 \% \mathrm{CI}: 0.64$ to 1.28 ). There was no difference in the reported number of cigarettes smoked during late pregnancy for participants classified at baseline as smokers (adjusted difference in means, FNP-Usual Care: 0.119 cigarettes, 97.5\% CI: -0.73 to 0.97$)$. On average, birth weights were marginally higher in the intervention arm compared to the control arm though this was not statistically significant (adjusted difference in means, FNP-Usual Care: 20.75 grams, 97.5\% CI: -47.73 to 89.23$)$. There was no difference in the proportion of women reporting a second pregnancy within the two years of their first child's birth (adjusted OR 1.01, 97.5\% CI: 0.77 to 1.33 ). Rates of child emergency attendances and admissions for any reason in secondary care prior to their second 
birthday were higher in the intervention arm though the difference was not statistically significant (adjusted OR 1.32, 97.5\% CI: 0.99 to 1.76). There were no differential effects due to age, deprivation, participation in employment, education or training, or basic life skills for any of the primary comparisons.

Secondary outcomes. Some benefit of the intervention was observed in terms of maternally rated language developmental delay in the children at both 12 (OR 0.50 , CI: 0.35 to 0.72 ) and 18 months (OR 0.66, CI: 0.48 to 0.90). This was further reflected in the Early Language Milestone assessment at the end of the trial period which showed higher scores on average for children in the intervention arm (adjusted difference of means, 4.49 , CI: 0.52 to 8.45 ) indicating more advanced levels of language development. Benefits of the intervention were also observed for breastfeeding intentions (adjusted OR 1.32, CI: 1.02 to 1.70 ) though this did not translate into a difference in the actual proportions of mothers initiating breastfeeding between the two arms.

A greater proportion of children in the intervention arm attended an Emergency Department for an injury or ingestion at 6 months (adjusted OR 1.52, CI 0.86 to 2.70) and 12 months (adjusted OR 1.16, CI 0.92 to 1.46), were referred to social services by two years (adjusted OR 1.27, CI: 0.93 to 1.73 ) and had a safe guarding event recorded in their GP record (adjusted OR 1.85, CI: 1.02 to 2.85) compared to children in the control group. These differences were not statistically significant.

No evidence of a difference was observed for any other maternal or parenting and child outcomes assessed in the trial.

At two years, mothers in the intervention arm reported lower rates of not being in employment, education or training (NEET) than the control arm, though this was not statistically significant. A larger proportion of participants in the intervention arms reported a maximum level of social support at 18 months postpartum $(25.7 \%)$ compared to those in the control arm $(20.3 \%)$ with a similar difference being maintained at 24 months. A small difference between the arms was observed over the whole follow-up period (adjusted OR 1.5, CI: 1.06 to 2.12). Fewer participants in the intervention arm reported ever being homeless during the study period compared to the control arm (adjusted OR 0.76, CI: 0.55 to 1.05) and higher levels of self-efficacy were observed in the intervention arm (adjusted mean difference 0.44 , CI: 0.10 to 0.78 ).

No evidence of a difference was observed for any other parental life course outcomes assessed in the trial.

\section{Discussion}

This analysis shows limited differences between the resource uptake and associated costs between the two trial arms (FNP intervention and usual care). As demonstrated in Table 2, for most of the health care resource use items, average costs were slightly lower for the FNP arm compared to usual care. Conversely resource use and associated average per person costs were higher for non-health related items. This however is not necessarily a negative outcome as some of the items associated with greater costs were positive aspects such as attendance at a mainstream education programme and attendance at children's centres. Despite these resources being considered positive outcomes it is important to remember that these are still additional costs which could be incurred as a result of the FNP intervention and should be considered in policy making. It is also worth noting that although there were more child A\&E attendances and admissions associated with families receiving the intervention, this is unlikely to be a detrimental effect of the intervention. Instead this probably reflects an increased awareness of mothers receiving the intervention, and their increased contact with a health professional could mean that health concerns are identified more readily causing mothers to seek more help.

Previous studies of the FNP programme in the United States (US) have shown positive results in terms of improved prenatal health, childhood injuries, subsequent pregnancies, maternal employment and child school readiness ${ }^{10-12}$. The Building Blocks study is the first randomised controlled trial of FNP in the UK. The statistically insignificant effects observed in the present work which are discussed in the effectiveness paper ${ }^{9}$ likely reflect the differences in health care provision between the US and the UK. In the US, low income mothers are not able to access many health care and social services. In contrast, all pregnant women in the UK can access a wide provision of maternal care including community care family doctors, midwives and specialist public health nurses. It is thus likely that the FNP programme does not offer a sufficiently enhanced service to the level of usual care provided in the UK. This study thus adds to the knowledge base of FNP and is useful to policy makers considering the value of implementing FNP within a UK-style health care system.

As a methodological approach, cost-consequence analysis is generally limited by its dependence on subjective decision making by policy makers, as it relies on individual judgement of what consequences are sufficiently beneficial to merit investment. The lack of a comparative statistical component could also be viewed as a limitation. However, the cost-consequence model is of value to provide a simplistic overview of costs associated with the intervention, particularly those that are not obvious such as youth offending services and family information services. A cost-consequence balance sheet was implemented to provide policy makers with a clear descriptive summary of the health and non-health related costs associated with the FNP intervention. This offers an advantage over more technical methods of economic analysis. Furthermore, though the use of QALYs is generally favoured by clinical decision making bodies such as the National Institute for Health and Care Excellence $(\mathrm{NICE})^{13}$, the calculation of QALYs may not always be sensitive in particular populations. When QALY gains were assessed in a cost-utility analysis conducted as part of the BB trial, no significant gains were identified and the intervention was deemed not cost-effective when considered from the perspective of the mother ${ }^{14}$. Given the dimensions of 
the EQ-5D (pain, mobility, self-care, usual activities, and depression/anxiety) it may be the case that the instrument was insensitive to the population and the intentions of the intervention to primarily improve child health outcomes rather than maternal health outcomes thus giving further weight to the case for considering outcomes beyond health.

The analysis considered only resource use of the mother; this is due to the overarching perspective of the wider economic analysis that was conducted alongside the trial, the primary analysis being a cost-utility analysis. Currently there is no accepted instrument for measuring utility in children as young as those in the Building Blocks trial hence the cost-utility analysis could only take the perspective of the mother. Future research could incorporate child resource use but given the absence of statistically significant results for any of the Building Blocks trial primary outcomes, this would not change the overall message of the work and hence was deemed unnecessary.

As an approach, cost-consequence analysis cannot usually conclude whether an intervention is worth funding as the judgement usually lies upon the gains that would likely be attained by recipients. However, given that the FNP intervention does not bring about any significant improvements to recipients compared to usual care on the basis of the short-term outcomes, together with the higher cost of delivering the intervention compared to usual care ( $£ 1812$ more per woman), these two factors suggest that based on current evidence the continuation of the programme cannot be justified.

In all, the cost-consequence model summarises the betweengroup differences in resource use and costs, alongside the primary trial outcomes. As can be seen, there are a large number of potential factors that are likely to attract additional costs beyond the obvious implementation costs of the intervention. Given these costs and the way in which only small benefits were observed in the trial analysis when considering primary outcomes alone, the cost-consequence model provides further support that the FNP intervention is unlikely to be worth the substantial costs and policy makers may wish to consider other options for investment.

\section{Ethical approval}

The trial is registered with International Standard Randomised Control Trial Number ISRCTN23019866 on 20 April 2009. The Building Blocks trial protocol and all amendments were reviewed and approved by the Wales NHS Research Ethics Committee (09/MRE09/08). All procedures were in accordance with the ethical standards set out in the 1964 Helsinki declaration and its later amendments.

\section{Data availability}

Underlying data

The Building Blocks Trial dataset comprises data from multiple sources including third party. The Hospital Episode Statistics (HES) data, which forms a substantial component of the present analysis, is supplied under licence from NHS Digital. The HES dataset is not currently available for analysis to researchers outside Cardiff (the lead institution) due to the information provided to participants at consent (which identified which institutions were acting as data controller / processors).

Researcher can apply for access to the full dataset by contacting Cardiff Centre for Trials Research (CTR) (see: https://www.cardiff.ac.uk/centre-for-trials-research/about-us/ data-requests). The restriction on HES data (originally supplied via NHS Digital) applies. CTR will consider whether section 251 approval would enable release of participant identifiers to apply directly to NHS Digital for the data used in the trial.

\section{Extended data}

The results of the Building Blocks trial are presented in full at: https://www.cardiff.ac.uk/_data/assets/pdf_file/0009/504729/ Building-Blocks-Full-Study-Report.pdf

This publication includes the full CONSORT statement for the trial on page 564 .

\section{Acknowledgments}

The South East Wales Trials Unit (SEWTU) is funded by the Wales Assembly Government through the Health and Care Research Wales and the authors gratefully acknowledge SEWTU's contribution to study implementation. We thank all the women who participated in the study, the local professionals who facilitated the recruitment and study implementation, and the family nurses who delivered the intervention. We acknowledge all the other contributors to the study who are listed in full study report. We thank all the trial steering committee independent members: Ann Louise Kinmonth (Chair), Silvia van den Heijkant, Pamela Park, Stavros Petrou, Rachel Tonkin; and the data monitoring committee independent members: Gordon Taylor (Chair), Lucy Akhtar, Sara Kenyon. We would like to pay special tribute to the late professor Paul Wainwright who has the initial Chair of the Data Monitoring Committee. We thank the stakeholder involvement work package members: Joyce Kenkre (lead), Lily Bidmead, Kamila Hawthorne, Lesley Lowes, with contributions from members of the Books \& Babies Group, and the Young Mums Groups. The trial administrators were Jackie Swain, Katy Addison, and Rhys Thomas.
1. Robling M, Bekkers MJ, Bell K et al: Effectiveness of a nurse-led intensive home-visitation programme for first-time teenage mothers (Building Blocks): a pragmatic randomised controlled trial. Lancet. 2016; 387(10014): 146-155. PubMed Abstract | Publisher Full Text | Free Full Text
2. National Institute for Health and Care Excellence: Methods for the Development of NICE Public Health Guidance [Internet]. (third edition), London: NICE. 2012. PubMed Abstract

3. Drummond M, Sculpher MJ, Torrance GW, et al.: Methods for the economic 
evaluation of health care programmes. 3rd edition, Oxford, UK: Oxford University Press, 2005

Reference Source

4. Brazier J, Ratcliffe J, Salomon J, et al.: Measuring and Valuing Health Benefits for Economic Evaluation. Oxford: Oxford University Press. 2007. Reference Source

5. Corbacho B, Bell K, Stamuli E, et al.: Cost-effectiveness of the Family Nurse Partnership (FNP) programme in England: Evidence from the building blocks trial. J Eval Clin Pract. 2017; 23(6): 1367-1374.

PubMed Abstract | Publisher Full Text

6. Department of Health: NHS Reference Costs 2012/13. 2013. Reference Source

7. Curtis L: Unit Costs of Health and Social Care 2013. Personal Social Services Research Unit: University of Kent. 2013. Reference Source

8. StataCorp: Stata Statistical Software: Release 12. StataCorp LP: College Station TX. 2011.

Reference Source

9. Robling M, Bekkers MJ, Bell K, et al:: Effectiveness of a nurse-led intensive home-visitation programme for first-time teenage mothers (Building Blocks): a pragmatic randomised controlled trial. Lancet. 2016; 387(10014): 146-155. PubMed Abstract | Publisher Full Text | Free Full Text

10. Kitzman $\mathrm{H}$, Olds DL, Henderson $\mathrm{CR}$ Jr, et al.: Effect of prenatal and infancy home visitation by nurses on pregnancy outcomes, childhood injuries, and repeated childbearing. A randomized controlled trial. JAMA. 1997; 278(8): 644-52. PubMed Abstract | Publisher Full Text

11. Kitzman $\mathrm{H}$, Olds DL, Sidora $\mathrm{K}$, et al:: Enduring effects of nurse home visitation on maternal life course: a 3-year follow-up of a randomized trial. JAMA. 2000; 283(15): 1983-9.

PubMed Abstract | Publisher Full Text

12. Olds DL, Kitzman $\mathrm{H}$, Hanks $\mathrm{C}$, et al.: Effects of nurse home visiting on maternal and child functioning: age-9 follow-up of a randomized trial. Pediatrics. 2007; 120(4): e832-45.

PubMed Abstract | Publisher Full Text | Free Full Text

13. National Institute for Health and Care Excellence: Guide to the methods of technology appraisal. London: NICE. 2013. Reference Source

14. Corbacho B, Bell K, Stamuli E, et al.: Cost-effectiveness of the Family Nurse Partnership (FNP) programme in England: Evidence from the building blocks trial. J Eval Clin Pract. 2017; 23(6): 1367-1374.

PubMed Abstract | Publisher Full Text 


\section{Open Peer Review}

\section{Current Peer Review Status:}

\section{Version 1}

Reviewer Report 08 October 2019

https://doi.org/10.5256/f1000research.22134.r53943

(C) 2019 Barnes J. This is an open access peer review report distributed under the terms of the Creative Commons Attribution License, which permits unrestricted use, distribution, and reproduction in any medium, provided the original work is properly cited.

\section{Jacqueline Barnes}

Department of Psychological Sciences, Birkbeck, University of London, London, UK

The Building Blocks team are to be congratulated for taking a cost-consequences approach (CCA) rather than the cost utility method relying on QALYs, the strategy frequently applied to interventions. The CCA is much more relevant for this type of trial, with many non-medical potential benefits included in the secondary outcomes. It is well known that the long-term impact of early intervention such as the Nurse Family Partnership (NFP, known as FNP in the UK) can be within the areas of education, employment or criminal justice. Unfortunately for the team they currently have information only up to the end of programme delivery when children were 24 months of age, and are reliant of the primary outcomes being medical in nature. Many of the most well documented impacts of the NFP intervention, from trials in the USA, have been identified when the children are in primary or secondary school, or during young adulthood. It is hoped that further follow-up of the trial participants will be feasible. The paper acknowledges this point.

The design is strong, albeit limited due to lack of sufficient costing of services used by infants, meaning that all of the services used are those use by the mother. Thus it is not clear whether a visit to a children's centre includes any services explicitly experienced only by the child are covered. One point about the costs applied to Children's Centre visits is that they are based on the economic evaluation of Sure Start Local Programmes (SSLPS). While Children's Centres, where if they still operate, are largely derived from SSLPs, the range of services is likely to be fewer meaning that costs may be in fact lower.

All the details of the method are clearly presented, meaning that the work could be reproduced if there was access to the full dataset. This is not possible since participant consent precluded anyone not a member of the Building Blocks team having access to the Hospital Episodes Statistics data. This is clearly stated in the paper.

The discussion is very sensible, noting that aspects of the mothers' experiences such as attending an educational establishment or a children's centre, while incurring costs at the time are likely to lead to less of a cost to society in the future if this leads to better employment prospects, or a child more ready to start school. Similarly more women and infants gaining access to housing rather 
than being homeless is of great benefit to society and to local communities. Thus it is disappointing that the paper concludes that continuation of the programme cannot be justified. This strong conclusion is not really warranted until the children can be followed until the beginning of formal education at the very least. The FNP children had some benefits in terms of language development so it is important to identify whether fewer required the support of speech and language therapists or educational psychologists and special education teachers. Thus it would have been useful to have some provisos included in the conclusion. While it is not possible in relation to the Building Blocks RCT, it would also be useful to comment that further research with a sample of women identified using additional risk indicators beyond age and first pregnancy, and one involving FNP practitioners with substantial experience rather than newly trained Family Nurses, would add to the capacity for commissioners to decide about continuing to support the FNP programme.

Is the work clearly and accurately presented and does it cite the current literature? Yes

Is the study design appropriate and is the work technically sound?

Yes

Are sufficient details of methods and analysis provided to allow replication by others? Yes

If applicable, is the statistical analysis and its interpretation appropriate? Yes

Are all the source data underlying the results available to ensure full reproducibility? Partly

Are the conclusions drawn adequately supported by the results? Partly

Competing Interests: No competing interests were disclosed.

Reviewer Expertise: Evaluation of programmes to support disadvantaged parents and children; The relevance of communities for parenting

I confirm that I have read this submission and believe that I have an appropriate level of expertise to confirm that it is of an acceptable scientific standard.

Reviewer Report 01 October 2019

https://doi.org/10.5256/f1000research.22134.r53944

(c) 2019 Hollingworth $\mathbf{W}$ et al. This is an open access peer review report distributed under the terms of the Creative Commons Attribution License, which permits unrestricted use, distribution, and reproduction in any medium, provided the original work is properly cited. 


\section{Shaun Harris}

Department of Population Health Sciences, Bristol Medical School, University of Bristol, Bristol, UK William Hollingworth

Department of Population Health Sciences, Bristol Medical School, University of Bristol, Bristol, UK

The authors present a cost-consequences analysis of a family nurse (FNP) led intervention (versus usual care) to support teenage mothers in England. The analysis is based on approximately 1600 participants recruited to the Building Blocks RCT. The randomised design and size of the study should provide robust results for policy makers.

Given the diverse range of costs and outcomes a cost consequence analysis is a reasonable and appropriate approach to take. The pragmatic approach to address missing data also appeared to be reasonable given the context. Key outcomes and cost-utility data from this trial have already been reported (Robling et al. 2016 ${ }^{1}$; Corbacho et al. 2017²). Therefore, the contribution of this paper is largely in providing additional details about health (and wider) resource use in each arm of the trial.

The results show that there is little difference in either resource uptake or associated costs between FNP and usual care. The authors conclude that the intervention is unlikely to be costeffective. The authors suggest that previous positive results from US studies might not be replicated in England due to more extensive existing access to maternity care in the UK.

There are several issues that should be clarified:

1. In Table 3, it would be useful to report the total healthcare costs to establish the aggregate cost differences (and 95\% CI) between FNP and usual care. These total costs are briefly mentioned on page 9 (paragraph 2) but do not appear in Table 3.

2. The cost of the FNP intervention itself should be included in Table 3. These are only introduced in the discussion section currently. Inclusion in Table 3 should allow the authors to estimate the net incremental healthcare costs of FNP.

3. Although there is no one agreed approach to inflating costs across difference sectors of the economy, the approach taken by the authors (of not standardizing cost years) seems unsatisfactory as it does not allow the estimation of total costs (healthcare and other sectors) that would be useful for readers/policy makers. A GDP deflator index - while not perfect - would be a better starting point.

4. Page 9 (paragraph 3): median values are not presented in table 3.

5. A brief description of the intervention theory and aims and the eligibility criteria would help this article 'stand alone' from previous articles.

\section{References}

1. Robling M, Bekkers MJ, Bell K, Butler CC, et al.: Effectiveness of a nurse-led intensive homevisitation programme for first-time teenage mothers (Building Blocks): a pragmatic randomised controlled trial.Lancet. 2016; 387 (10014): 146-55 PubMed Abstract | Publisher Full Text 
2. Corbacho B, Bell K, Stamuli E, Richardson G, et al.: Cost-effectiveness of the Family Nurse Partnership (FNP) programme in England: Evidence from the building blocks trial.J Eval Clin Pract. 2017; 23 (6): 1367-1374 PubMed Abstract | Publisher Full Text

Is the work clearly and accurately presented and does it cite the current literature? Yes

Is the study design appropriate and is the work technically sound? Yes

Are sufficient details of methods and analysis provided to allow replication by others? Partly

If applicable, is the statistical analysis and its interpretation appropriate?

Partly

Are all the source data underlying the results available to ensure full reproducibility? Partly

Are the conclusions drawn adequately supported by the results? Yes

Competing Interests: No competing interests were disclosed.

Reviewer Expertise: Health Economics

We confirm that we have read this submission and believe that we have an appropriate level of expertise to confirm that it is of an acceptable scientific standard.

The benefits of publishing with F1000Research:

- Your article is published within days, with no editorial bias

- You can publish traditional articles, null/negative results, case reports, data notes and more

- The peer review process is transparent and collaborative

- Your article is indexed in PubMed after passing peer review

- Dedicated customer support at every stage

For pre-submission enquiries, contact research@f1000.com 